\title{
LA EMPRESA SOCIAL DE MUHAMMAD YUNUS, UN NUEVO PARADIGMA PARA ERRADICAR LA POBREZA
}

\author{
MUHAMMAD YUNUS' SOCIAL BUSINESS, A NEW PARADIGM TO ERADICATE \\ POVERTY
}

René Mora Casal ${ }^{1}$

\begin{abstract}
Resumen
La empresa social representa un nuevo paradigma para erradicar la pobreza en el mundo, dentro de un modelo de mercado que respeta la dignidad humana. Se analiza esta innovación social con base en los escritos de su creador y principal promotor, Muhammad Yunus, Premio Nobel de la Paz 2006. Asimismo, se contrastan estas ideas con las de otros autores que han analizado el tema de la pobreza (tales como Bornstein, Prahalad, Sachs, De Soto), algunos de los cuales adoptan el punto de vista de la economía tradicional, cuyas ideas y métodos han sido criticados por Muhammad Yunus. Finalmente, se hace una reflexión sobre la relatividad del concepto de pobreza y la obligación moral de combatirla.
\end{abstract}

Palabras clave: Empresa social; Muhammad Yunus; pobreza; innovación social; capitalismo social.

\begin{abstract}
Social business represents a new paradigm to eradicate world poverty, within a market model that respects human dignity. This social innovation is analyzed based on its creator and main promoter, Muhammad Yunus, 2006 Nobel Peace Prize winner. In addition, these ideas are contrasted with those of other authors who have studied the topic of poverty (such as Bornstein, Prahalad Sachs, De Soto). Some of them have adopted the point of view of traditional Economics and have been criticized by Muhammad Yunus for their ideas and
\end{abstract}

doi: http://dx.doi.org/10.15359/eys.20-47.3

Fecha de recepción: 2-11-2014. Fechas de reenvíos: 7-03-2015/ 03-06-2015. Fecha de aceptación: 22-06-2015. Fecha de publicación 29-06-2015.

1 Doctor en Ingeniería Química, Atlantic International University. Estados Unidos. Máster en Administración de Negocios, Universidad Interamericana de Costa Rica. Licenciado en Ingeniería Química, Universidad de Costa Rica. Consultor privado, Costa Rica. Correo electrónico morarene1@gmail.com 
methods. Finally, the author reflects on relative poverty and the moral obligation to fight it.

Key words: Social business; Muhammad Yunus; poverty; social innovation; social capitalism.

\section{Introducción}

TOMS es una empresa fundada en 2006 por Blake Mycoskie, con dos propósitos: obtener un beneficio económico y usar este beneficio para una causa social. El Programa One for One de TOMS dona un par de zapatos a una persona en condición de pobreza en un país menos desarrollado por cada par que vende: hasta la fecha ha donado 35 millones de pares de zapatos $^{2}$. Este es un ejemplo de una empresa social, y el presente ensayo tiene por objeto analizar este concepto y su potencial para reducir la pobreza en el mundo, a partir de la obra Building Social Business: The New Kind of Capitalism That Serves Humanity's Most Pressing Needs, de Muhammad Yunus, fundador del Banco Grameen y Premio Nobel de la Paz 2006. Dicho libro está estrechamente relacionado con las obras anteriores del mismo autor, así como con el desarrollo de los microcréditos a nivel mundial, concepto que él acuñó y al que se hará referencia como ejemplo de innovación social. Asimismo, se contrastarán estas ideas para el combate de la pobreza en el mundo con puntos de vista alternativos al de Yunus.

\section{¿Quién es Muhammad Yunus?}

Muhammad Yunus es un economista de Bangladesh, país asiático ubicado entre los más pobres del mundo, quien fue galardonado con el Premio Nobel de la Paz 2006 "por sus esfuerzos para incentivar el desarrollo social y económico desde abajo", según se indica en el sitio de la organización, www.nobelprize.org. Uno de los aportes de Yunus es la implementación del concepto de microcrédito en Bangladesh y otros países menos desarrollados a partir de 1974, lo que llevó a la fundación del Banco Grameen (aldea en su idioma natal) en 1983. Hoy los microcréditos se han extendido por todo el mundo y son un medio demostrado para ayudar a las personas a salir de la pobreza extrema mediante el fomento del espíritu emprendedor y de la dignidad humana: para el 2006 se habían otorgado microcréditos a 100 millones de prestatarios, lo cual benefició a un estimado de 500 millones de personas. Para el año 2015 se espera haber incrementado estas cifras en un 75\% (Spiegel, 2007, pp. 62,108-109).

El microcrédito se basa en la idea de que un pequeño préstamo, de entre 20 y 30 dólares estadounidenses, puede ser suficiente para que una persona pobre inicie un negocio y pueda salir de la trampa de la pobreza (Spiegel, 2007, p. 29). En este sentido, se puede afirmar que el

${ }^{2}$ Información disponible en el sitio web de la empresa, www.toms.com

2

René Mora Casal 
Banco Grameen funciona principalmente como una banca para el desarrollo. Los principios en los que se basa el modelo de trabajo del Banco Grameen son opuestos a los de la banca tradicional, pero no al capitalismo: orientación hacia los más pobres y hacia las mujeres, no se piden garantías, se evita la burocracia, un sistema basado en la transparencia y la confianza. Como resultado, este banco ha obtenido una tasa de devolución de créditos del 99 \% (Spiegel, 2007, pp. 29-55). Los resultados de los microcréditos en Bangladesh y otros países han sido evidentes: mayor acceso a la educación, mejora en la condición de las mujeres, reducción de la tasa de natalidad, mejores condiciones de vivienda y salud, aumento del nivel de salarios y mayor estabilidad político-social (Spiegel, 2007, pp. 86-101).

En forma paralela, mediante la Fundación Grameen, se han creado múltiples empresas que brindan servicios en Bangladesh con una orientación social y empresarial a la vez (Spiegel, 2007, pp. 137-145), lo que ha llevado a Yunus a desarrollar el concepto de empresa social, su segundo aporte y tema central del presente ensayo. Yunus cree firmemente que es posible erradicar la pobreza, desterrarla a los museos, a tal punto de afirmar que esta existe porque la hemos aceptado en nuestros esquemas mentales y que no será hasta que la pobreza se vuelva inaceptable que empezaremos a ver cambios en el mundo (Spiegel, 2007, pp. 103, 152; Yunus, 2008).

\section{Yunus y las empresas sociales}

El último libro de Muhammad Yunus, titulado Building Social Business: The New Kind of Capitalism That Serves Humanity's Most Pressing Needs (en español Las empresas sociales: una nueva dimensión del capitalismo para atender las necesidades más acuciantes de la humanidad) es una continuación de los temas tratados en su libro anterior, del año 2008, Creating a World Without Poverty: Social Business and the Future of Capitalism (en español Un mundo sin pobreza: las empresas sociales y el futuro del capitalismo), donde se introduce el concepto de empresa social y la historia del desarrollo de la empresa Grameen-Danone, cuyo fin es elaborar yogur fortificado y a bajo costo para los niños de Bangladesh. El mismo Banco Grameen es un ejemplo de empresa social, el cual desde 1995 no acepta fondos no reembolsables, solo de inversionistas (Spiegel, 2007, p. 81). Desde el 2013 el Banco Grameen ha recibido 885 propuestas para proyectos de empresas sociales solo en Bangladesh, de los cuales han aprobado 860 y muchas de estas empresas ya están en operación, como lo reporta el mismo Profesor Yunus en su página web, www. muhammadyunus.org.

En un sentido amplio, empresa y emprender se definen como "acción o tarea que entraña dificultad y cuya ejecución requiere decisión y esfuerzo." (Real Academia Española, 2012). Una empresa social -según Yunus- es una organización creada con el objetivo primario de resolver un problema social, ambiental, sanitario o similar, y con el objetivo paralelo y secundario de generar ingresos suficientes para ser sostenible en el tiempo; la maximización de ganancias y la satisfacción de los accionistas, principios rectores de la empresa capitalista tradicional, están fuera de este concepto (Yunus, 2010, 
p. 1). Esta organización es diferente de los programas de caridad o de responsabilidad social pues tiene otros principios y objetivos: de hecho Yunus ha criticado el papel de estos programas en la lucha por erradicar la pobreza, al igual que las actuaciones del gobierno, de los bancos y de los organismos internacionales (Spiegel, 2007, pp. 56-59, 99, 109-112).

Yunus distingue dos tipos de empresas sociales: la empresa que no busca el lucro, sino resolver un problema social, y la empresa que es propiedad de personas pobres y que les permite generar ingresos así como mejorar su condición y la de su comunidad (Yunus, 2010, pp. 1-2). Asimismo, establece los siguientes siete principios que caracterizan a la empresa social (Yunus, 2010, p. 3):

1. El objetivo de la empresa es superar la pobreza o resolver uno o más problemas sociales, no el de maximizar las ganancias.

2. La empresa debe ser sostenible financiera y económicamente.

3. Los inversionistas recuperan solo la inversión inicial.

4. Cuando se devuelve la inversión, las ganancias se reinvierten en la empresa para ampliación y mejoras; es decir, no hay dividendos.

5. La compañía será ecológicamente responsable.

6. Los empleados de la empresa tienen un salario y condiciones de trabajo iguales o mejores que el estándar local.

7. ¡Este trabajo se hace con alegría!

Los principios (3) y (4) anteriores son interesantes por ser contrarios al pensamiento tradicional, donde el inversionista busca precisamente los dividendos o una ganancia sobre su inversión; también cabe mencionar la pérdida de valor del dinero con el tiempo debido a la inflación y otros factores. Más adelante en su libro, Yunus escribe sobre la importancia de crear fondos de inversión social e incluso una bolsa de valores social, iniciativas que necesariamente están asociadas con algún tipo de compensación para los inversionistas, lo cual parece contradictorio con lo anterior: se puede proponer que esta compensación sea la mínima necesaria para que el dinero no pierda su valor.

Los principios (5) y (6) no son de carácter económico o financiero, sino que están motivados por el carácter social de la empresa, en particular dado que el capitalismo tradicional, tipo laissez faire o "salvaje", ha generado explotación laboral, accidentes laborales y contaminación ambiental en todo el mundo, tal como se practica en China actualmente (Ohmae, 2008). En cuanto a los salarios, esta iniciativa es similar a los programas de Fair Trade mediante los cuales se les paga a los agricultores un precio justo por sus productos, superior al precio de mercado: el tema de la justicia es clave en ambos casos. El último principio es de carácter psicológico, importante, ya que el trabajo en una empresa social responde a una vocación que está más enfocada en la realización personal mediante el servicio a los demás que en la obtención de poder o de bienes materiales.

4 
La empresa social de Yunus es un ejemplo de aplicación del Pensamiento Lateral ${ }^{3}$ (De Bono, 1970), pues se toma un concepto tradicional como la banca y se cambia completamente, puesto que se crean los microcréditos y el Banco Grameen. En este sentido Yunus es un genio social ${ }^{4}$, lo que le ha permitido extender sus ideas por el mundo; también ha sido calificado recientemente como un innovador financiero y un pionero de los negocios por la revista Financial Times (Mackintosh, 2015).

Asimismo, la empresa social es un ejemplo de lo que Steven Covey llama la tercera alternativa: bajo este concepto se analiza una situación con dos polos u opciones, por ejemplo "Pobreza: Justicia social vs. Responsabilidad personal" ${ }^{5}$, y se establece un compromiso de buscar una alternativa de carácter superior que sea aceptada por todos y que sea beneficiosa para ambas partes, es decir una situación del tipo "ganar-ganar" (Covey, 2012). Yunus ha afirmado que la caridad o la asistencia social pueden ser tan dañinas como no hacer nada, ya que "la limosna es la mayor ofensa que se le puede hacer a un pobre” (Spiegel, 2007, pp. 80, 83, 153).

Muhammad Yunus combina en una forma destacable la innovación social con la ortodoxia económica: la empresa social debe cubrir sus costos, hacer un plan de negocios, buscar y atraer socios e inversionistas, tener una estrategia empresarial y una administración sólidas, mercadearse, etc. (Yunus, 2010). Este autor cree en un capitalismo con rostro humano que sirva a fines más altos que solamente la obtención de riquezas; por ejemplo, en el caso de los microcréditos, un principio establecido por el Banco Grameen es que nunca se condona un préstamo, aún en situaciones extremas como pérdidas de cosechas, incendios, inundaciones $u$ otras; lo que se hace es adecuar el plazo y las condiciones del préstamo. Esta "rigidez" corresponde en el fondo a un principio ético: el de tratar a las personas pobres como clientes emprendedores y no como mendigos, lo cual ha producido resultados muy efectivos (Spiegel, 2007, pp. 75-82).

En el año 2010 se publicó un artículo en la revista Long Range Planning sobre las lecciones adquiridas por el Grupo Grameen en la creación de empresas sociales. En dicho artículo se plantea un modelo para construir una empresa social, la cual debe tener necesariamente los siguientes componentes (Yunus, Moingeon y Lehmann-Ortega, 2010):

Una propuesta de valor: responde a la pregunta "¿Quiénes son nuestros clientes y cuáles aspectos valoran de los productos que les ofrecemos?".

\footnotetext{
${ }^{3}$ Técnica que busca la solución de un problema mediante métodos indirectos y no ortodoxos, con un enfoque creativo. Fue creada por Edward de Bono en 1967.

${ }^{4}$ Así califica Howard Gardner a Gandhi en su libro Mentes creativas (Gardner, 1998). También se puede afirmar que Yunus posee una elevada inteligencia emocional (ver Goleman, 1995).

${ }^{5}$ Esta es la dicotomía que plantea Covey, de las que surge una tercera alternativa que él llama "Dignidad humana".
} 
- Una constelación de valor, formada por la cadena de valor de la compañía y por la red de valor externa que posee, responde a la pregunta “¿Cómo entregamos nuestra oferta de valor a nuestros clientes y accionistas?".

- Una ecuación de ganancias, de carácter financiero y que explica cómo se emplea el capital, cómo es la estructura de costos y cómo se captura el valor mediante los ingresos generados.

- A lo anterior se debe agregar una ecuación de ganancia social, que es característica de la empresa social y no existe en las empresas tradicionales, en ella se establece las ganancias o el valor de tipo tanto social como ambiental que se van a generar mediante la actividad o negocio.

Otros elementos que se deben tener en cuenta según este artículo son: considerar a todas las partes interesadas en la propuesta y en la constelación de valor y no solo a los clientes y accionistas; definir claramente las ganancias sociales que se persiguen y excluir la maximización de las utilidades de la ecuación de ganancias, y así mantener el objetivo relacionado con que no haya pérdida económica (Yunus, Moingeon y Lehmann-Ortega, 2010).

Muhammad Yunus escribe en su libro acerca de cinco lecciones que ha aprendido en su trabajo de creación de empresas sociales (Yunus, 2010, pp. 50-53):

1. Sea flexible pero nunca pierda de vista el objetivo central -y social- de su negocio.

2. Sumérjase en la cultura de la gente a la cual usted intenta servir -el incumplimiento de esto es la principal crítica de Yunus a los gobiernos y a los asesores económicos internacionales-.

3. Utilice la ayuda de aliados donde quiera que los pueda encontrar.

4. Aproveche las ventajas de las diferentes oportunidades que se presentan en distintos mercados.

5. Periódicamente revise y cuestione las suposiciones de su modelo de negocio.

Además de Grameen-Danone y del Banco Grameen, el libro presenta varios ejemplos de empresas sociales, la mayoría en Bangladesh y relacionados con el grupo Grameen, entre ellas (Yunus, 2010): la Fundación Cure2Children (atención de talasemia en sur de Asia); GrameenVeolia Water (agua potable a bajo costo); el Laboratorio Creativo Grameen (difusión de la experiencia y conocimiento del Grupo Grameen); BASF-Grameen (toldos para repeler los mosquitos, empaques con micronutrientes para niños); Grameen-Intel (salud materno-infantil) y Grameen-Adidas (calzado a costo mínimo).

Yunus menciona también en su libro que las empresas sociales se han convertido en objeto de estudio en universidades alrededor del mundo, con el fin de establecerlas en sus respectivos países. Gobiernos de países como Colombia y Haití están aplicando este concepto para resolver sus problemas más apremiantes; asimismo, las empresas sociales están generando interés por parte de

6

René Mora Casal

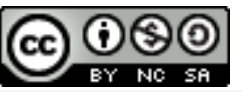

Revista Economía y Sociedad by Universidad Nacional is licensed under a Creative Commons Reconocimiento-NoComercialCompartirlgual 4.0 Internacional License. Creado a partir de la obra en http://www.revistas.una.ac.cr/index.php/economia 
inversionistas y de fondos de inversión, con la expectativa de que en el futuro próximo se establezca una Bolsa de Valores solo para estas empresas. El mensaje final y principal de este libro es que el cambio sí es posible, y que mediante las empresas sociales sería posible erradicar la pobreza en este siglo (Yunus, 2010).

En general, este libro ha sido recibido en forma favorable por sus lectores, pero sin considerarlo excelente como evidencia su calificación de 3,92 de 5 estrellas en el sitio www.goodreads.com, basada en 583 calificaciones. En los círculos especializados las ideas de este libro han sido criticadas con argumentos - discutibles en mayor o menor grado- tales como:

- Que la definición de empresa social de Yunus es rígida y dogmática, poco razonable, dejando por fuera muchas empresas con elementos sociales como cooperativas, ONG y otras (Schwartz, 2010; Kaminsky, 2010).

- Que al requerir que no tengan lucro limita sus posibilidades de crecimiento y sus fuentes de capital de inversión, haciéndolas dependientes de empresas multinacionales (Schwartz, 2010; Hausmann, 2010; Kaminsky, 2010).

- Escepticismo con respecto a la sostenibilidad de estas empresas, así como a que vayan a transformar el mundo, pues es una idea en pañales y cuyo éxito todavía debe ser demostrado (Esquinca, 2009; Leonard, 2010).

- Yunus no inventó el microcrédito ni las empresas sociales, y pinta una caricatura de las empresas capitalistas (Hausmann, 2010); de hecho el concepto de empresa social tiene más de cien años (Schwartz, 2010).

- Las empresas Tipo II de Yunus, que sí buscan el lucro pero que tienen como dueños a personas pobres, ¿dejan de ser sociales si tienen éxito? (Schwartz, 2010).

- Estas empresas no pueden competir con otras de mayor capital; pueden producir competencia desleal y afectar los salarios de trabajadores de otras empresas; serían una amenaza para empresas competidoras; habría que diseñar un sistema para que solo los pobres compraran sus productos o servicios (Esquinca, 2009) ${ }^{6}$.

- En el Capítulo 1 de su libro, Yunus dice que las organizaciones sin fines de lucro dependen de donaciones caritativas o filantrópicas y por eso no son empresas sociales. En nuestra opinión esto es un error conceptual, pues en realidad existen diversas formas en que se financian estas organizaciones.

\section{Contraste de ideas: Yunus versus otros autores}

Algunos de los críticos de Muhammad Yunus afirman que él no inventó el concepto de empresa social, o bien dudan de que sirva para erradicar la pobreza; por tanto conviene buscar los

${ }^{6}$ Esta es tal vez la crítica más discutible. En Bangladesh, debido a la sustracción de mano de obra de la fuerza laboral, en pocos años el salario obrero se multiplicó por cinco (Spiegel, 2007, p. 94). 
paralelismos y las diferencias que tiene el pensamiento de Yunus con respecto al de otros autores que han investigado o bien trabajado activamente en el tema, con el fin de probar la originalidad del primero -o su falta de ella-. Este contraste de ideas se puede desarrollar con la ayuda de una serie de conceptos planteados por los autores seleccionados: emprendimiento social, riqueza en la base de la pirámide, el fin de la pobreza, el misterio del capital.

\section{Emprendimiento social}

Se puede afirmar que el concepto de empresa social (social business) no fue inventado por Muhammad Yunus, aunque él así lo cree cuando dice "una empresa social es un nuevo tipo de negocio" (Yunus, 2010, p. 1). En Inglaterra del siglo XIX se habían creado las cooperativas y otros tipos de organizaciones sin fines de lucro y con un claro objetivo social (Schwartz, 2010); en los últimos treinta años han surgido miles de organizaciones sin fines de lucro, grupos ciudadanos, empresas sociales y emprendedores sociales en todo el mundo (Bornstein, 2004).

Las personas que crean una empresa social pueden ser llamadas emprendedores sociales (Ashoka, s.f.). Ejemplos históricos de emprendedores sociales han sido: Florence Nightingale (fundadora de la Enfermería moderna), María Montessori (nuevo método de educación de niños), John Muir (creación de los primeros parques nacionales). Un ejemplo moderno de empresa social -no relacionada con Muhammad Yunus- es la fundación Ashoka, que desde el año 1981 apoya a emprendedores sociales y se ha extendido en 70 países alrededor del mundo (Ashoka, s. f., Bornstein, 2004); otro ejemplo es la empresa TOMS mencionada al inicio. En el año 2011, la revista Forbes elaboró una lista de los 30 emprendedores sociales más destacados del mundo (Coster, 2011).

Los emprendedores sociales más exitosos comparten seis características (Bornstein, 2004, cap. 18), que se pueden considerar complementarias a las cinco lecciones de Yunus:

- Están dispuestos a admitir sus errores y corregir el rumbo.

- Están dispuestos a compartir el crédito de sus logros.

- Están dispuestos a enfrentar y romper las estructuras existentes.

- Crean equipos interdisciplinarios para resolver problemas.

- Trabajan calladamente, sin buscar reconocimiento.

- Tienen un alto sentido ético, el dinero es una herramienta para unos fines más elevados.

\section{Riqueza en la base de la pirámide}

C. K. Prahalad, en su libro La oportunidad de negocios en la base de la pirámide, señala que las empresas privadas deberían crear productos y servicios adaptados para atender a la mayoría de la población mundial (4 000 millones de personas) que forman la "base de la pirámide" y viven con USD 2 o menos por día, pero que en conjunto representan un potencial inmenso de

8

René Mora Casal

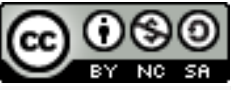

Revista Economía y Sociedad by Universidad Nacional is licensed under a Creative Commons Reconocimiento-NoComercialCompartirlgual 4.0 Internacional License. Creado a partir de la obra en http://www.revistas.una.ac.cr/index.php/economia 
negocio. En estos mercados, particularmente en países como India y China, el bajo precio se vería compensado por la enorme cantidad de clientes potenciales, lo que aseguraría un negocio rentable. El libro explica ampliamente las pautas y principios para que las empresas desarrollen negocios en el tercer mundo (por ejemplo, adaptarlos para personas analfabetas y que no sean contaminantes).

Un concepto importante que menciona Prahalad es el de "sanción por pobreza", es decir, que los bienes y servicios básicos son más caros para las personas pobres, por ejemplo tienen que pagar tasas de entre $600 \%$ y $1000 \%$ a los prestamistas locales (Prahalad, 2006, pp. 17-18). Este último hecho fue uno de los motivos que impulsó a Muhammad Yunus a iniciar el proyecto que luego llegó a convertirse en el Banco Grameen (Spiegel, 2007; pp. 25-29).

Prahalad propone modelos de negocio basados en unidades pequeñas, bajo precio, márgenes bajos de utilidad, altos volúmenes y alto rendimiento sobre el capital (Prahalad, 2006, p. 36). Esto se ilustra con el caso de Grameen Danone: cuando se duplicó el precio de la leche en abril del 2008, primero aumentaron el precio, lo que resultó ser un error; la solución fue reducir el tamaño de la unidad (Yunus, 2010, pp. 45-49). Prahalad reconoce que el consumo por parte de la población más pobre puede traer asociados problemas ambientales serios, por ejemplo en temas como los empaques desechados y el consumo de agua; por eso recomienda que las soluciones deben ser sostenibles y benignas para el ambiente (Prahalad, 2006, pp. 39-40, 51-53, 89-90).

Prahalad presenta, en la segunda parte de su libro, varios ejemplos exitosos de empresas que se pueden considerar empresas sociales, de acuerdo -en todo o en parte- con la definición de Yunus: Casas Bahia, Sal Annapurna, Jaipur Foot, Voxiva. Asimismo, ejemplos de empresas privadas grandes que han desarrollado líneas de negocios dirigidas a la población pobre, como el proyecto Patrimonio Hoy de CEMEX en México.

Prahalad considera que hay varias razones por las que la población pobre se verá beneficiada por este enfoque, tales como: liberación de su creatividad y espíritu empresarial; acceso a mejores productos y servicios y, por tanto, a una mejor calidad de vida; reducir o eliminar la sanción por pobreza; eliminar barreras de comunicación; acceso al conocimiento; reconocimiento legal e identidad; mejora en la condición de la mujer; reducción de la corrupción (Prahalad, 2006, pp. 151-170). La visión de Prahalad es poder llegar a convertir la pirámide en un diamante (p. 167). Si se hace una comparación con las ideas de Muhammad Yunus, se observa que, aunque los enfoques son distintos, los efectos "sociales" que se buscan son similares. Por otra parte, Prahalad no explica cómo este consumo (o consumismo) va a beneficiar a los que viven en extrema pobreza, tema que no se toca en su libro.

El libro de Prahalad fue declarado el mejor libro de negocios del 2004 por la revista Fast Company y por www.amazon.com, y uno de los mejores libros publicados en 2004 por la revista 
The Economist; sin embargo no ha estado exento de críticas. La crítica más demoledora a la "proposición de la base de la pirámide" proviene de un colega de Prahalad en la Universidad de Michigan, Kaneel Arnani, quien desmantela las premisas y los ejemplos que sustentan este modelo -se sobrestima el tamaño del mercado, los costos de distribución son muy altos, los ejemplos exitosos no se dirigen realmente a la población pobre, es incorrecto comparar precios de tercer mundo con los del primer mundo, se han creado problemas ambientales, algunos negocios realmente explotan a la persona pobre, entre otros-, y concluye que se trata de "un milagro" en el mejor caso y de "una ilusión peligrosa" en el peor (Karnani, 2006). En otro artículo, Karnani indica que la visión "romántica" del pobre como emprendedor y consumidor racional es dañina, pues motiva a quitarle la protección que realmente necesita por parte del Estado y del sistema legal y de seguridad social (Karnani, 2007). La solución real para Karnani es aumentar el ingreso real de las personas pobres, ya sea proporcionándoles empleos de calidad o bien considerándolos como productores y no como consumidores (Karnani, 2006). Karnani también ha criticado los microcréditos, pues afirma que en su mayor parte solo generan empresas y empleos de subsistencia (Karnani 2006, 2007).

\section{El fin de la pobreza}

Jeffrey Sachs es un economista estadounidense, asesor de las Naciones Unidas y de gobiernos alrededor del mundo, especialista en temas de pobreza y crisis económicas. En el año 2006 publica su libro El fin de la pobreza: cómo conseguirlo en nuestro tiempo, donde hace una propuesta para erradicar la pobreza extrema en el mundo antes del año 2025, definida esta como un nivel de ingresos inferior a un dólar de los EE. UU. por día.

Muhammad Yunus y Jeffrey Sachs presentan varias semejanzas entre ellos: ambos son economistas que creen en la capacidad del capitalismo para generar desarrollo y reducir la pobreza, un capitalismo con sentido social; los dos parten del hecho de que la salida de la pobreza comienza con la capacidad para ahorrar y reinvertir recursos (Sachs, 2006, pp. 92-94; Spiegel, 2007, p. 34); tanto Yunus como Sachs han sido críticos del papel que han jugado los organismos internacionales, como el FMI y el Banco Mundial, a la hora de brindar ayuda o asistencia a los países pobres, en donde se condicionan muchas veces dicha ayuda a determinadas políticas macroeconómicas (Sachs, 2006, pp. 126-132); asimismo han criticado que la mayor parte de dichas ayudas se destinan al pago de estudios y de los salarios de asesores y burócratas, quienes muestran poca sensibilidad hacia los más pobres (Sachs, 2006, pp. 374, 416-417, 432; Spiegel, 2007, pp. 56-59). Sachs promueve una "economía clínica" que conozca profundamente las características particulares del país (Sachs, 2006, pp. 121-140), lo cual coincide con las ideas de Yunus de adaptarse a las necesidades de los pobres y no al revés (Spiegel, 2007, pp. 24-25, 85-86).

Por otra parte, también hay diferencias importantes entre los enfoques y las propuestas de Yunus y de Sachs:

10

René Mora Casal

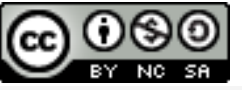

Revista Economía y Sociedad by Universidad Nacional is licensed under a Creative Commons Reconocimiento-NoComercialCompartirlgual 4.0 Internacional License.

Creado a partir de la obra en http://www.revistas.una.ac.cr/index.php/economia 
- Yunus propone un mundo sin pobreza; Sachs propone erradicar la pobreza extrema para que los países más pobres puedan iniciar "su propia ascensión por la escalera del desarrollo económico" (Sachs, 2006, p. 56).

- Yunus promueve el microcrédito y la empresa social para acabar con la pobreza; Sachs promueve la liberalización económica en conjunto con una asistencia económica enfocada en el cumplimiento de los Objetivos del Milenio de las Naciones Unidas (ODS).

- Sachs promueve que la ayuda de los países desarrollados a los países más pobres se incremente hasta un 0,7 \% de su Producto Nacional Bruto anualmente, hasta que se cumplan los ODS (Sachs, 2006, pp. 310, 416-421). Yunus, en cambio, no está de acuerdo con el enfoque asistencialista, pues considera que esto equivale a una limosna, "la mayor ofensa para un pobre" (Spiegel, 2007, p. 83). Yunus solo acepta la ayuda financiera en situaciones de emergencia como incendios, inundaciones y hambrunas (Spiegel, 2007, p. 76).

- Yunus aplica los principios del capitalismo, pero no dudó en revisar las prácticas bancarias tradicionales y hacer lo opuesto si era necesario (Spiegel, 2007, p. 12). Por el contrario, Sachs utilizó las herramientas económicas tradicionales para ayudar a resolver las crisis en varios países: control de la inflación, recompra de bonos, liberalización y privatización, fondos de estabilización, entre otras, e incluso reconoce que su propuesta de condonar las deudas de los países más pobres proviene de una propuesta similar que hizo J. M. Keynes en 1919 para los países europeos (Sachs, 2006, pp. 156-157).

\section{El misterio del capital}

Hernando De Soto es un autor mencionado tanto por Peter Spiegel en su biografía de Muhammad Yunus, como por C. K. Prahalad y Jeffrey Sachs en sus respectivos libros. Este economista peruano realizó estudios sobre las economías informales alrededor del mundo y vertió sus conclusiones en varios libros, el más importante de ellos es El misterio del capital del año 2002. Según este autor, los pobres tienen recursos enormes en forma de activos diversos (terrenos, herramientas, animales, etc.), el problema es que son recursos informales. Para poder cambiar esta situación, De Soto propone regularizar la tenencia de propiedad de los activos de las personas pobres, esto les permitiría ser sujetos de crédito para generar negocios y salir de la pobreza. Lo anterior no va a ser posible si en el país existe una pesada burocracia, por lo que también se debe trabajar en agilizar los trámites locales (Spiegel, 2007, pp. 31-32).

Esta idea ha sido ensayada en países como Brasil; sin embargo, los bancos siguen sin aceptar a los pobres como sujetos de crédito, aunque sean dueños de los activos, debido a un prejuicio social (Fernandes, 2002a). Las ideas de Hernando De Soto han sido criticadas por otros académicos, quienes opinan que la pobreza no se debe a un único factor, sino a muchos (Fernandes, 2002b; Sachs, 2006, pp. 446-447). 
Una diferencia importante entre las visiones de Hernando de Soto y de Muhammad Yunus es que el primero es un economista tradicional, para quien los bancos no le prestan a una persona si no tiene garantías reales; en cambio, Yunus demostró con la creación del Banco Grameen que esto es un prejuicio, que no se requieren garantías de ningún tipo para otorgarle préstamos a las personas más pobres y que se puede diseñar un sistema de tipo bancario basado en la confianza, con un fin social y a la vez sostenible financieramente.

\section{Reflexiones sobre el tema de la pobreza}

Se ha presentado el pensamiento y la labor de diferentes personas que en algún momento reflexionaron sobre la pobreza en el mundo y llegaron a soluciones específicas, como son las empresas sociales promovidas por Muhammad Yunus, los emprendimientos sociales de la Fundación Ashoka y los Objetivos del Milenio de las Naciones Unidas que impulsa Jeffrey Sachs. Sin embargo, cabe preguntarse qué ha motivado a estas personas a preocuparse -y ocuparsepor los que menos tienen, los que están más abajo en la escala social. Para los propósitos del presente ensayo, se proponen tres preguntas generadoras con el fin de explorar esto:

- ¿Qué es ser pobre? ${ }^{7}$

- ¿Ser pobre es una condición siempre negativa, indeseable?

- ¿Estamos moralmente obligados a resolver la pobreza?

Con respecto a la primera pregunta, se puede afirmar que existe tanto pobreza absoluta como pobreza relativa. Los economistas (ej. Banco Mundial) manejan actualmente una definición absoluta de pobreza moderada asociada con un ingreso diario de USD 2 por día y de pobreza extrema asociado con un ingreso de USD 1,25 al día ${ }^{8}$ basados en una paridad del poder de compra a precios del 2005 (Banco Mundial, 2008); este es el concepto de pobreza que se ha venido discutiendo en este ensayo. Por otra parte, la pobreza relativa es un concepto social: Jeffrey Sachs ilustra lo anterior en su libro señalando que los pobres de los países desarrollados serían considerados clase media en los países más pobres (Sachs, 2006, p. 49). Como ejemplo de esto, el Instituto Nacional de Estadística y Censos reportó en el Censo 2000 que el $79 \%$ de los tugurios en Costa Rica -que es un país de renta media- tenían televisor en color, lo cual implica que también tenían electricidad (Leitón, 2002); asimismo, en el Censo 2011 se reportó que el $71,5 \%$ de los tugurios tenían al menos un teléfono celular (Arias, 2011). Esto se explica como un asunto de necesidades básicas frente a otras necesidades (la escala de Maslow), las primeras

\footnotetext{
7 Estas preguntas se formulan desde un punto de vista personal, el de un costarricense que durante su niñez y juventud vivió en condiciones modestas, podríamos decir de pobreza relativa, pero que sin embargo nunca se sintió "pobre" porque siempre vio cubiertas sus necesidades básicas, desde ropa y alimentación hasta su educación y apoyo emocional de su familia y su comunidad.

${ }^{8}$ Previamente la línea de pobreza extrema se definía como USD 1 al día (ver p. ej. Sachs, 2006).
}

12

René Mora Casal

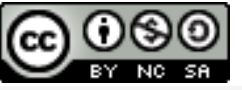

Revista Economía y Sociedad by Universidad Nacional is licensed under a Creative Commons Reconocimiento-NoComercialCompartirlgual 4.0 Internacional License. Creado a partir de la obra en http://www.revistas.una.ac.cr/index.php/economia 
son cubiertas por las latas o los cartones, mientras que las segundas son satisfechas por el televisor o el teléfono celular.

El ejemplo contrario proviene del área del mercadeo: la publicidad nos hace sentirnos "pobres" si no tenemos un "teléfono inteligente" u otros artículos; vemos entonces el caso de personas humildes que compran cosas que no necesitan, que es una de las críticas que hace Aneel Karnani al libro de Prahalad (Karnani, 2006, pp. 14-19).

A pesar de lo relativo del concepto "pobreza", existe un nivel absoluto que debe considerarse intolerable y que es la pobreza extrema, asociada con el hambre, la desnutrición y las enfermedades, y que mata a millones de personas cada año ${ }^{9}$.

En cuanto a la segunda pregunta, la pobreza moderada no necesariamente tiene una connotación negativa, pues una persona puede ser pobre materialmente, pero puede tener una riqueza interior de tipo cultural, artística o espiritual. Muchas de las manifestaciones culturales y artísticas en el mundo, desde la música y las leyendas folklóricas hasta las catedrales medievales de Europa, fueron el producto de personas pobres. También la comunidad puede ser una red de apoyo importante para aliviar la carga de la pobreza.

Otras personas han elegido ser pobres por razones filosóficas o religiosas, por ejemplo los filósofos Sócrates y Diógenes en la Grecia antigua, o Francisco de Asís en la Italia del siglo XII, este último seguidor de otro pobre como fue Jesús de Nazaret. Un ejemplo moderno existe en el estado de Utah (EE. UU.), donde vive Daniel Suelo, quien desde el año 2000 renunció a todo tipo de dinero por razones tanto filosóficas como religiosas, como lo relata Mark Sundeeen en el libro The Man Who Quit Money. Daniel Suelo es una persona destacable porque vive en una cueva y busca su comida en los basureros, pero a la vez es un miembro activo de su comunidad y tiene un blog, por lo que no calza con la imagen tradicional del "pobre" desvalido (Sundeen, 2012).

La tercera pregunta tiene por sí misma múltiples respuestas, según la ideología y las motivaciones de cada persona u organización. Por ejemplo, la mayoría de las religiones del mundo enseñan que el auxiliar a los pobres es una obligación moral, pero esto no necesariamente significa sacarlos de la pobreza. Algunas personas y organizaciones ayudan a los pobres porque está bien visto socialmente hacerlo, mientras que otras inventan razones para no ayudarlos. Se puede incluso considerar ventajoso que existan pobres en el mundo, porque son "servidumbre" o "mano de obra barata", y por tanto hay que dejarlos en su situación actual. Muhammad Yunus sintió una obligación moral hacia las personas pobres, pero consideró que

\footnotetext{
${ }^{9}$ Hay otros elementos asociados con la pobreza, como son la delincuencia, la drogadicción, la explotación, la ira, la violencia, etc. que tampoco son tolerables, pero que están fuera de los alcances de este ensayo.
} 
son capaces de rescatarse ellas mismas y que solo requieren una ayuda inicial, tal como hace el Banco Grameen en Bangladesh.

En Costa Rica existen personas que han sentido o sienten una obligación moral de ayudar a las personas pobres (incluido este autor), y se han creado programas estatales de asistencia social para reducir la pobreza; sin embargo, en los últimos veinte años la desigualdad social ha venido aumentando mientras que los porcentajes de pobreza (moderada y extrema) se han mantenido, lo cual implica un aumento de la cantidad de pobres en términos absolutos (Arias et al., 2011; Ramón, 2015). El hecho que Uruguay, un país con un nivel de renta y otras características similares a las de Costa Rica, tenga para el año 2014 niveles de pobreza del 6,4 \% y de pobreza extrema del 0,2 \% mientras que en nuestro país estos valores son de $22,4 \%$ y 5,8 \% son una muestra del trabajo pendiente por realizar (Rodríguez \& Leitón, 2014; Instituto Nacional de Estadísticas, 2015).

Las reflexiones anteriores pueden servir de motivación para definir qué tipo de empresas sociales podemos desarrollar en Costa Rica, y qué tipo de problemas son los que deben ser resueltos o qué proyectos vale la pena desarrollar, por ejemplo:

- Combatir la desnutrición infantil.

- Enseñar hábitos de higiene y prevención de enfermedades.

- Actividades educativas artísticas y deportivas para niños, jóvenes y adultos.

- Educación de adultos en habilidades para el trabajo.

- Educación de adultos en liderazgo.

- Programas de prevención de la violencia familiar.

- Programas de prevención de la drogadicción.

- Programas de reciclaje de desechos sólidos.

- Programas de limpieza de playas y ríos, entre otros.

La propuesta de Muhammad Yunus constituye un nuevo paradigma que puede transformar "desde adentro" las vidas de las personas más pobres del mundo, puesto que se reconocen sus capacidades y se atienden sus necesidades reales. Asimismo, los profesionales de Costa Rica y del mundo (incluso este autor) pueden, mediante la creación de empresas sociales en sus respectivos países, encontrar la realización personal y darse cuenta que esa persona pobre es otro ser humano, su "prójimo".

A manera de conclusión, les aporto dos anécdotas personales. La primera ocurre en diciembre del 2013, cuando este autor visitó la ciudad de Chicago, una de las más grandes y hermosas de los Estados Unidos, quedó impresionado al ver personas pobres que pedían limosna a lo largo de la "Milla Magnífica" en el frío invierno. El comentario a su esposa en ese momento, y que quedó grabado en su mente, fue: "Debe ser terrible ser pobre en Chicago".

14

René Mora Casal

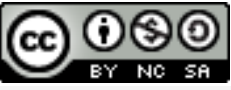

Revista Economía y Sociedad by Universidad Nacional is licensed under a Creative Commons Reconocimiento-NoComercialCompartirlgual 4.0 Internacional License. Creado a partir de la obra en http://www.revistas.una.ac.cr/index.php/economia 
La segunda ocurrió hace más de treinta años, cuando este autor era un niño, y la escuchó de boca de un misionero católico estadounidense: él y su grupo llegaron a un pueblo muy pobre en la zona rural de Costa Rica y le preguntaron a la gente: “¿Qué quieren que les construyamos?". La gente no pidió una escuela, un templo, o un dispensario médico, como era de suponer: pidió una pista de baile, que los misioneros construyeron. Al año siguiente, cuando ellos regresaron, la gente del pueblo ya había construido por su propia iniciativa la escuela, el templo y el dispensario médico. ¡Eso es trabajo con alegría!

\section{Referencias}

Arias, R.; Sánchez, L.; Sánchez, R. (2011). Análisis de la desigualdad socioeconómica en Costa Rica. Revista Economía y Sociedad, 39-40(16), 73-107. Recuperado de http://www.revistas.una.ac.cr/index.php/economia/article/view/4902

Ashoka (s.f.). What is a Social Entrepreneur? Recuperado de https://www.ashoka.org/social entrepreneur

Ashoka (s. f.). About Us. Recuperado de https://www.ashoka.org/about

Banco Mundial (16 de septiembre de 2008). New Data Show 1.4 Billion Live On Less Than US\$1.25 A Day, But Progress Against Poverty Remains Strong. Press Release. Recuperado de http://www.worldbank.org/en/news/press-release/2008/09/16/newdata-show-14-billion-live-less-us125-day-progress-against-poverty-remains-strong

Bornstein, D. (2004). How to Change the World: Social Entrepreneurs and the Power of New Ideas. Oxford: Oxford University Press. Recuperado de https://books.google.co.cr/books/about/How to Change the World Social Entrepre n.html?id=P g8gVyuuEgC\&redir esc=y

Coster, H. (2011). How We Selected the Forbes Impact 30 List. Revista Forbes. Recuperado de http://www.forbes.com/sites/helencoster/2011/11/30/impact-30-methodology/

Covey, S. (2012). La tercera alternativa. Barcelona: Paidós. Recuperado de https://books.google.co.cr/books/about/La 3a alternativa.html?id=nVbJVuCwsXYC\&re dir esc=y 
De Bono, E. (1970). Lateral Thinking: Creativity Step by Step. New York: Harper \& Row. Recuperado https://books.google.co.cr/books/about/Lateral thinking_creativity step by step.htm ? $\mathrm{id}=\mathrm{H}-\mathrm{ROAAAAMAAJ} \&$ redir_esc=y

Esquinca-Barriga, E. S. (2009). La empresa social: ¿cura o paliativo para la pobreza? Revista Confines, 9, 115-118. Recuperado de http://confines.mty.itesm.mx/articulos9/EsquincaE.pdf

Fernandes, E. (Julio 2002a). Políticas de suelo en América Latina/Entrevistador: Martim Smolka. Lincoln Institute of Land Policy. Recuperado de http://www.lincolninst.edu/pubs/762 Perfil-Docente--Edesio-Fernandes

Fernandes, E. (2002b). The Influence of de Soto's The Mystery of Capital. Lands Lines, 1(14). Recuperado de http://www.lincolninst.edu/pubs/199 Land-Lines--January-2002-Volume-14--Number-1

Gardner, H. (1998). Mentes creativas: una anatomía de la creatividad. Barcelona: Paidós. Recuperado de https://books.google.co.cr/books/about/Mentes_creativas.html?id=GuewOwAACAAJ\& redir esc=

Goleman, D. (1995). La inteligencia emocional. Santiago de Chile: Editorial Vergara.

Hausmann, R. (2010). To Profit or Not to Profit: Is That the Question?. Finance and Development, 2(47). Recuperado http://www.imf.org/external/pubs/ft/fandd/2010/06/books.htm

Instituto Nacional de Estadística. (2015). Estimación de la pobreza por el método del ingreso, año 2014. Montevideo: INE. Recuperado de http://www.ine.gub.uy/biblioteca/pobreza/Pobreza\%202014/Informe\%20Pobreza\%20 2014.pdf

Kaminsky, P. (2010). Building Social Business. Alternatives Journal. Recuperado de: http://www.alternativesjournal.ca/community/reviews/building-social-business

Karnani, A. (2006). Fortune at the Bottom of the Pyramid: A Mirage (Working Paper Series, No. 1035). Retrieved from the web site of the University of Michigan Ross School of Business: http://deepblue.lib.umich.edu/bitstream/handle/2027.42/41223/1035?sequence $=5$

16

René Mora Casal

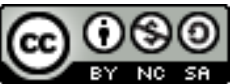

Revista Economía y Sociedad by Universidad Nacional is licensed under a Creative Commons Reconocimiento-NoComercialCompartirlgual 4.0 Internacional License. Creado a partir de la obra en http://www.revistas.una.ac.cr/index.php/economia 
Karnani, A. (2007). Romanticing the Poor Harms the Poor (Working Paper Series, No. 1096). Retrieved from web site of the University of Michigan Ross School of Business: http://deepblue.lib.umich.edu/bitstream/handle/2027.42/55470/1096Karnani.pdf?sequence $=1 \&$ isAllowed $=y$

Leitón, P. (25 de marzo de 2002). "Tele" y "refri" dominan en casas ticas. Diario La Nación. Recuperado de http://www.nacion.com/economia/Tele-refri-dominan-casasticas 0 527947221.html

Leonard, D. (1 de mayo de 2010). Microcredit? To Him, It's Only a Start. New York Times. Recuperado de http://www.nytimes.com/2010/05/02/business/02shelf.html? $r=0$

Ohmae, K. (2008). El próximo escenario global: desafíos y oportunidades en un mundo sin fronteras. Bogotá: Editorial Norma S.A. https://books.google.co.cr/books/about/El Pr\%C3\%B3ximo escenario global.html?id= bPUGol-USNYC\&redir esc=y

Prahalad, C.K. (2006). La oportunidad de negocios en la base de la pirámide. Bogotá: Grupo Editorial Norma. Recuperado de https://goo.gl/Sw0dXf

Ramón, G. R. (26 de enero de 2015). Costa Rica se estanca en reducción de la pobreza y la desigualdad. La Nación. Recuperado de http://www.nacion.com/economia/Costa-Ricaestanca-reduccion-desigualdad 0 1465853516.html

Real Academia Española (2012). Diccionario de la lengua española (22a. ed.). Recuperado de http://lema.rae.es/drae/

Rodríguez, O.; Leitón, P. (30 de octubre de 2014). Pobreza en Costa Rica llega al nivel más alto en cinco años. La Nación. Recuperado de http://www.nacion.com/economia/politicaeconomica/hogares-Costa-Rica-pobres_0 1448255302.html

Sachs, J. (2006). El fin de la pobreza: cómo conseguirlo en nuestro tiempo. México D.F: Editorial Debate. Recuperado

de: https://books.google.co.cr/books/about/El fin de la_pobreza.html?id=F3lJPQAACAAJ \&redir esc $=y$

Schwartz, R. (2010). Hear, Hear for Profits. Standford Social Innovation Review, 4(8). Recuperado de http://ssir.org/issue/fall 2010 
Spiegel, P. (2007). Muhammad Yunus, el banquero de los pobres. Santander: Sal Terrae. Recuperado de http://www.casadellibro.com/libro-muhammad-yunus-el-banquero-delos-pobres/9788429317152/1150502

Sundeen, M. (2012). The Man Who Quit Money. New York: Riverhead. Recuperado de https://docs.google.com/document/d/1rkQjQpTPNXvbyvUQOMLAD3F3p36RZ3p1LkBmXwKfe4/edit

Yunus, M.; Moingreau, B.; Lehmann-Ortega L. (2010). Building Social Business Models: Lessons from the Grameen Experience. Long Range Planning (2-3) 43, 308-325. Recuperado de http://www.sciencedirect.com/science/journal/00246301/43/2-3

Yunus, M. (2008). Creating a World Without Poverty: Social Business and the Future of Capitalism. New York: Public Affairs. Recuperado de http://www.publicaffairsbooks.com/book/paperback/creating-a-world-withoutpoverty/9781586486679

Yunus, M. (2010). Building Social Business: The New Kind of Capitalism That Serves Humanity's Most Pressing Needs. New York: Public Affairs. Recuperado de http://www.publicaffairsbooks.com/book/paperback/building-socialbusiness/9781586489564

18 